\title{
The Quality of Tuberculosis Services in Health Care Centres in a Rural District in Uganda: The Providers' and Clients' Perspective
}

\author{
Lilian Bulage, ${ }^{1,2,3}$ Juliet Sekandi, ${ }^{1,4}$ Omar Kigenyi, ${ }^{1}$ and Ezekiel Mupere ${ }^{5}$ \\ ${ }^{1}$ Makerere University, College of Health Sciences, School of Public Health-Kampala, Uganda \\ ${ }^{2}$ National Tuberculosis and Leprosy Programme-Kampala, Uganda \\ ${ }^{3}$ National Tuberculosis Reference Laboratory-Kampala, Uganda \\ ${ }^{4}$ College of Public health, University of Georgia, 101 Buck Road, Athens, GA 30602, USA \\ ${ }^{5}$ Department of Paediatrics \& Child Health College of Health Sciences, Makerere University-Kampala, Uganda
}

Correspondence should be addressed to Lilian Bulage; lilian.bulage@gmail.com

Received 12 March 2014; Revised 7 August 2014; Accepted 12 August 2014; Published 7 September 2014

Academic Editor: Vincent Jarlier

Copyright (C) 2014 Lilian Bulage et al. This is an open access article distributed under the Creative Commons Attribution License, which permits unrestricted use, distribution, and reproduction in any medium, provided the original work is properly cited.

Quality of care plays an important role in the status of tuberculosis (TB) control, by influencing timely diagnosis, treatment adherence, and treatment completion. In this study, we aimed at establishing the quality of TB service care in Kamuli district health care centres using Donabedian structure, process, and outcomes model of health care. A cross-sectional study was conducted in 8 health care facilities, among 20 health care workers and 392 patients. Data was obtained using face-to-face interviews, an observation guide, a check list, and record review of the TB unit and laboratory registers. Data entry and analysis were done using EPI INFO 2008 and STATA 10 versions, respectively. A high number 150 (87.21\%) of TB patients were not aware of all the signs to stop TB medication, and $100(25.51 \%)$ patients received laboratory results after a period of 3-5 working days. The major challenges faced by health workers were poor attitude of fellow health workers, patients defaulting treatment, and fear of being infected with TB. One of the worst performance indicators was low percentage of cure. Comprehensive strengthening of the health system focusing on quality of support supervisions, patient follow up, promoting infection control measures, and increasing health staffing levels at health facilities is crucial.

\section{Introduction}

Tuberculosis $(\mathrm{TB})$ remains a major public health problem worldwide. There were 8.6 million new TB cases and 1.3 million TB deaths in 2012 [1]. The African region alone accounted for $27 \%$ of the world's cases and the highest rates of cases and deaths relative to population (225 incident cases per 100,000 on average and more than double the global average of 122) [1].

Uganda's TB burden remains unacceptably high, ranking 19th among the 22 high burden TB countries globally. In 2010, Uganda only reached $57 \%$ case detection rate (CDR) as opposed to the WHO target of $70 \%$. Kamuli, a rural district located in the South Eastern TB Zone as designated by the
Uganda National TB and Leprosy Program (NTLP), had $35.7 \%$ CDR for new smear positive cases. Out of the 225 sputum smear positive cases captured in 2010, 84 (37.3\%) got cured, 106 (47.1\%) completed treatment, 13 (6\%) died, $1(0.4 \%)$ was a failure, $16(7.1 \%)$ defaulted treatment, and the other 5 (2.2\%) were transferred to another district (Kamuli Annual Report on TB/Leprosy Control 2011).

Literature suggests that poor quality of care may result from discrepancies in documentation such as underreporting and gaps in the continuum of care services that the patients receive [1]. Donabedian developed the structures, process, and outcomes model as a framework for assessing the quality of health care services [2]. Structure consists of physical health facility, medical equipment, and staff characteristics. 
Processes of care involve interactions between users and the health care structures, the actual delivery, and receipt of care. Outcomes are consequences of care. Structures as well as processes may influence outcome, indirectly or directly [3]. There is low comprehensive knowledge about TB in the general population including lack of information on the TB service delivery points among other factors [4].

In Uganda, TB health care services at the district level are delivered through a continuum of various health facility structures designated as hospitals and Health Centers IV and III. Depending on the structural level of the health facility, the processes of care may begin with patient screening by health providers using specific case screening tools like the desk aids, intensified TB case finding forms (ICF), and the suspect register. The screening process could happen at outpatient clinics, specialized HIV/AIDS clinics, waiting rooms, and/or in-patient wards. The TB suspects are then registered and referred to the laboratory where bacteriological diagnosis is done on two samples (one spot and one early morning) by either Ziehl Neelsen (ZN) or fluorescence microscopy and documentation is done in the laboratory $\mathrm{TB}$ register [5]. At all the TB service points, the TB suspects or patients are supposed to be given specific information about TB symptoms, diagnosis, treatment, and follow up. Confirmed TB patients are registered and information is transferred to the health unit TB register. Patients are started on antituberculosis treatment, monitored monthly while on treatment for clinical and bacteriologic response, and evaluated at the end of treatment to document the relevant outcomes.

Given the various points within the health services that have to be traversed, the services patients receive can be compromised at the structural process or outcome levels leading to poor quality of TB care. However, the contributing structural and process factors have not been systematically studied and documented. Therefore, this study sought to establish the factors that influence quality of services among new smear positive TB cases in health care centers in Kamuli district. We adapted the Donabedian's structure, process, and outcomes model as the conceptual framework to study the factors that influence quality of TB services (Figure 1).

The results can inform the NTLP about the status of the quality of TB services in this rural setting and highlight pragmatic ways in which services can be improved.

\section{Methods}

2.1. Study Design. A cross-sectional study was conducted in Kamuli district using mixed methods. Both qualitative and quantitative data were collected. The patients' overall perception of the services together with the WHO, NTLP, and International Standards for Tuberculosis Care (ISTC) were used to judge the overall quality of services offered at the facilities. Quality was measured by the difference between expected and actual performance to identify opportunities for improvement.

2.2. Study Area. Kamuli district located in the South Eastern TB Zone of Uganda, $150 \mathrm{kms}$ from Kampala, was the study area selected on the basis of having poor TB outcomes for the new sputum smear positive cases in the year 2010. The district has 2 hospitals, 2 health center fours (HCIV), and 11 health centre threes (HCIII). Malaria, acute respiratory infections, intestinal worms, sexually transmitted infections (STI), and HIV and AIDS are the commonest causes of morbidity in the district.

2.3. Study Population. The study was carried out at tuberculosis diagnostic and treatment health units and the study participants were health unit in-charges, in-charges antiretroviral therapy (ART) clinics, TB focal persons, TB suspects, and confirmed TB patients aged 15 years and above and found at the health facilities on the day of visit. The above health workers were selected based on the knowledge base they had on TB services and logistics for TB care.

2.4. Inclusion and Exclusion Criteria. Diagnostic treatment facilities were included in the study. All the health workers (HW) who participated had worked at the facility for at least 3 and above months. Any TB suspects aged 15 years and above who had gone through all the service points up to the level of getting results from the laboratory, confirmed TB cases who had gone up to the point of receiving treatment or were already on treatment and found at the health facility at the time of the visit were included in the study. Patients too weak to be interviewed as judged by the research assistant were excluded and transfer ins from the health facility treatment register were also excluded from both the record review and interviews.

2.5. Sample Size. To establish the quality of the structural and processes of TB service care, 20 health workers and 392 (determined using the Leslie Kish formula) patients were interviewed after accounting for a $10 \%$ nonresponse rate. The number of patients to be interviewed at each facility was determined using the probability proportionate to size method. Kamuli Government hospital 182, Kamuli Mission Hospital 69, Namwendwa HCIV 53, Bulopa HCIII 10, Balawoli HCIII 32, Butansi $16 \mathrm{HCIII}$, and Bupadhengo HCIII had 30 patients interviewed. There were no patient interviews in Nankandulo HCIV, because at that time laboratory TB diagnosis had been stopped though we went ahead to interview the health workers. To establish the level of TB service care outcomes, all the study facilities were included and data for the new smear positive patients captured in 2010 was reviewed.

2.6. Sampling Procedure. A list of all the diagnostic treatment units (DTU) was obtained from the district health officer (DHO) to ensure that a representative number was considered for the study. From the list, the 2 hospitals (Kamuli Government Hospital and Kamuli Mission Hospital), 2 HCIV (Nankandulo HCIV and Namwendwa HCIV) were considered. However, since the HCIIIs were many, 2 high work load (Balawoli HCIII and Bupadhengo HCIII) and 2 low work load health facilities (Butansi HCIII and Bulopa HCIII) were considered for the study. Selection of health workers was done 


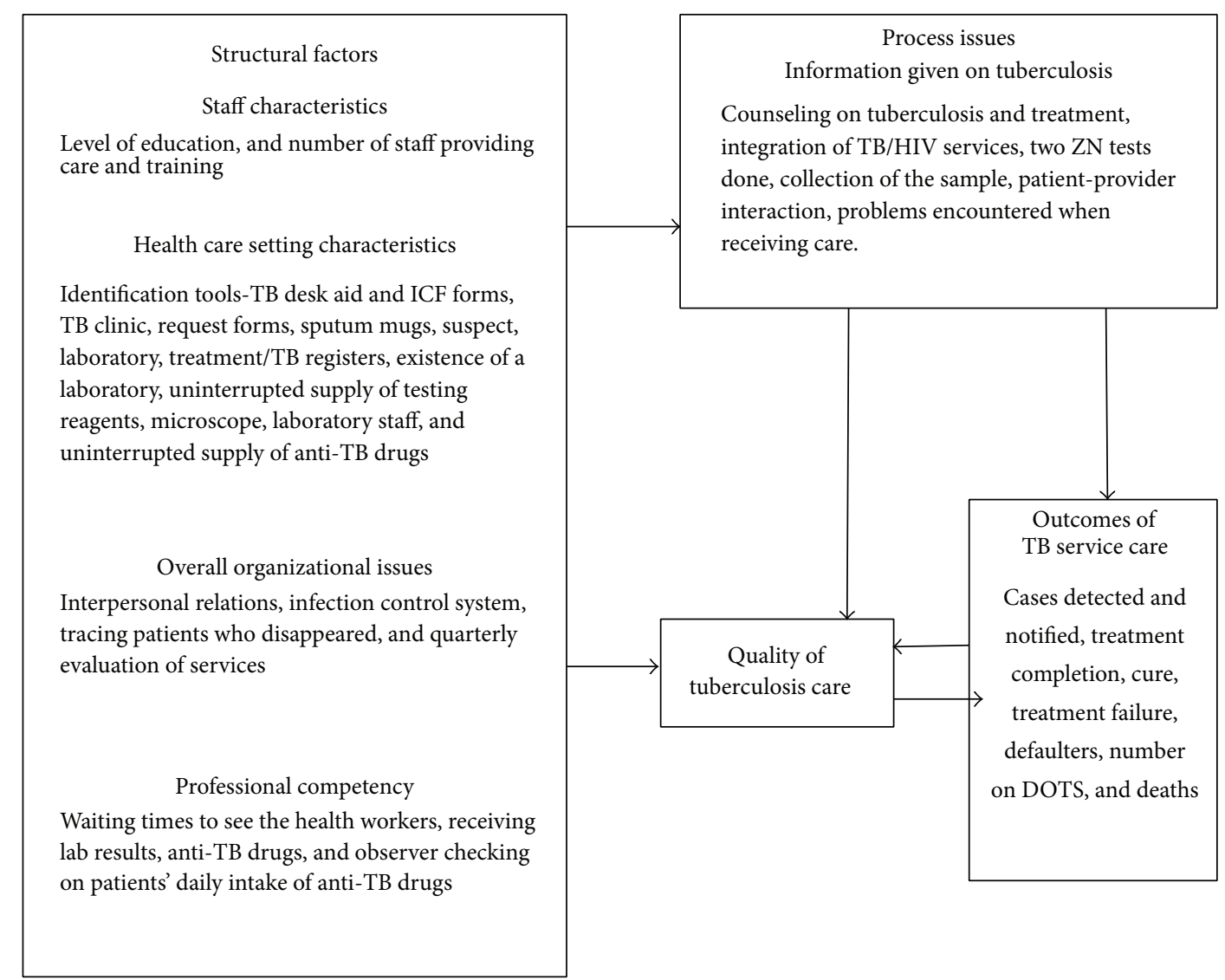

FIGURE 1: Conceptual frame work for quality of TB service in Kamuli district: adopted from the Donabedian model of quality of health care.

purposively. Tuberculosis patients (suspects or TB cases) exiting were enrolled to participate in the study consecutively until the required sample size was got. Data recorded in the TB laboratory and unit TB register for the year 2010 were reviewed to collect information on the number of $\mathrm{ZN}$ smears, cases notified, treatment completion, cure, defaulting, and death.

2.7. Data Collection. Information on quality of the structural and process factors was got by use of an on-site observation guide, a checklist, and holding face to face interviews using a semistructured questionnaire with in-charges of health care units, in-charges ART clinics, and TB focal persons. Tuberculosis suspects and cases were too face-to-face interviewed on the quality of process, some structural aspects, and overall rating of the facility in terms of quality. Data in the TB laboratory register was also reviewed to get information on the number of suspects assessed and how many of the ZN tests had been done for each suspect for the year 2010 . Information on outcomes of $\mathrm{TB}$ care was got by reviewing records in the health unit treatment $\mathrm{TB}$ registers for the year 2010 since it was expected that all the patients who were detected and started on treatment in that year had already had an outcome. To judge the overall quality of a particular health facility, the information collected about the quality of structural, process, and level of outcomes of TB service care was summarized into an indicator table which eventually led to a final judgment of the quality of services offered. Least performance score implied good quality services while the greatest score implied poor quality services offered by a facility (Table 1). The best and worst quality performance facilities and indicators were identified.

2.8. Quality Control. Two research assistants with knowledge on TB care were recruited for each facility and trained for 2 days on issues concerning this study. The research assistants were both English and Lusoga speakers whose least level of education was ordinary level.

2.9. Data Management. Data was entered and checked using EPI INFO 2008 version and exported to STATA version 10 for analysis. Missing data was ignored since it was small compared to the total data set count. Univariate analysis was run to obtain the baseline characteristics of the patients. Univariate analysis was also done to establish proportions, frequencies, and percentages regarding the performance of the health units on the different parameters of TB service delivery. For confidentiality reasons, the health facility names were replaced with letters when reporting on the number of $\mathrm{ZN}$ tests done, the TB outcomes, and the general quality performance of each facility. In order to clearly indicate the most serious and common problems faced by health care 
TABLE 1: Performance indicators/guidelines against which quality for Kamuli district health facilities was judged.

\begin{tabular}{|c|c|c|c|}
\hline $\begin{array}{l}\text { Health facility expected performance } \\
\text { indicators or guidelines }\end{array}$ & Actual performance level & Source of data & Performance score \\
\hline \multicolumn{4}{|c|}{ Structural performance guidelines/indicators } \\
\hline $\begin{array}{l}\text { All health workers participate in } \\
\text { identification of TB suspects }\end{array}$ & $\begin{array}{l}1=\text { Yes } \\
2=\mathrm{No}\end{array}$ & $\begin{array}{l}\text { Interviewed health facility } \\
\text { heads, TB focal persons, } \\
\text { and in-charges ART }\end{array}$ & $\begin{array}{l}1=\text { good } \\
2=\text { poor }\end{array}$ \\
\hline $\begin{array}{l}\text { Waiting times for; (i) Receiving } \\
\text { laboratory results } \\
\text { (ii) Receiving treatment }\end{array}$ & $\begin{array}{l}\text { (i) } 1=24 \text { hours } \\
2=>24 \text { hours }(50 \% \text { and above } \\
\text { of clients getting results after } \\
24 \text { hours) } \\
\text { (ii) } 1=\text { immediate } \\
2=>1 \text { day }\end{array}$ & Interviewed patients & $\begin{array}{l}1=\text { good } \\
2=\text { poor }\end{array}$ \\
\hline
\end{tabular}

Process performance guidelines/indicators

Two sputum samples should be collected for diagnosis

All suspects should have two ZN smears done

$1=$ Yes

$2=$ No

Percentage of TB suspects who were assessed by the laboratory with at least one $\mathrm{ZN}$ test

All TB suspects should be assessed using a symptom based approach

To assess and foster adherence to treatment, a patient-centered approach to administration of drug treatment, based on the patient's needs and mutual respect between the patient and the provider, should be developed for all patients. Supervision and support should be individualized and should draw on the full range of recommended interventions and available support services, including patient counseling and education

HIV testing and counseling should be recommended to all patients with, or suspected of having, tuberculosis Each healthcare facility caring for patients who have, or are suspected of having, infectious tuberculosis should develop and implement an appropriate tuberculosis infection control plan

Cured

Treatment failures

Defaulters

Died
Each TB positive patients should be on community based-DOTs
Every TB suspect should be advised to test for HIV

Presence of a system of identifying coughing patients from waiting areas and other places
Percentage declared cured

Number declared as failures

Percentage defaulters, no patient should default

Percentage died, no patient is expected to die
Reviewed laboratory register data for 2010

$1=81-100 \%=$ good

$2=70-80 \%=$ fair

$3=<70 \%=$ poor

Interviewed health facility heads, in-charges ART, and TB focal persons

$1=\operatorname{good}$

$2=$ poor

Reviewed the laboratory register and identified patients who were registered but no test done for them at all
$1=0$ patients $=$ very good

$2=1-3$ patients $=$ good

$3=4 \&>$ patients $=$ poor
Reviewed the unit TB treatment register
$1=$ all patients $=$ good

$2=$ some patients not on DOTs $=$ poor

Process performance guidelines/indicators

Interview with patients

Interviewed health facility heads, in-charges ART, and TB focal persons
$1=$ all patients $=$ good

$2=$ some patients not advised $=$ poor

$1=\operatorname{good}$

$2=$ poor

Outcome performance guidelines/indicators

Reviewed data in the treatment register for 2010

Reviewed data in the treatment register for 2010

Reviewed data in the treatment registers for 2010

Reviewed data in the treatment register for 2010
$1=85-100 \%=$ good

$2=$ less than $85 \%=$ poor

$1=0$ patients $=$ good

$2=1$ or $>$ patients $=$ poor

$1=0$ patients $=$ good

$2=1-7$ patients $=$ fair

$3=8$ and $>$ patients $=$ poor

$1=0=$ good

$2=1-5$ patients $=$ fair

$3=5$ and $>$ patients $=$ poor 
TABLE 2: Baseline characteristics of the patients attending health facilities in Kamuli district, March-May 2012.

\begin{tabular}{|c|c|c|}
\hline Characteristic & Frequency $(N=392)$ & Percentage \\
\hline \multicolumn{3}{|l|}{ Sex } \\
\hline Male & 165 & 42.20 \\
\hline Female & 226 & 58.80 \\
\hline \multicolumn{3}{|l|}{ Type of patient } \\
\hline Suspects & 220 & 56.12 \\
\hline Newly confirmed TB cases & 33 & 8.42 \\
\hline $\mathrm{TB}$ cases on treatment & 139 & 35.46 \\
\hline \multicolumn{3}{|l|}{ Age category } \\
\hline $15-24$ & 62 & 15.82 \\
\hline $25-34$ & 123 & 31.38 \\
\hline $35-44$ & 128 & 32.65 \\
\hline $45-80$ & 79 & 20.15 \\
\hline \multicolumn{3}{|l|}{ Residence } \\
\hline Rural & 294 & 75.0 \\
\hline Urban & 98 & 25.0 \\
\hline \multicolumn{3}{|l|}{ Occupation } \\
\hline Peasant farmer & 249 & 63.52 \\
\hline Civil servant & 26 & 6.63 \\
\hline Business & 81 & 20.66 \\
\hline Students & 27 & 6.89 \\
\hline Others $^{1}$ & 9 & 2.30 \\
\hline \multicolumn{3}{|l|}{ Highest education attained } \\
\hline None & 69 & 17.60 \\
\hline Primary & 191 & 48.72 \\
\hline Secondary & 108 & 27.55 \\
\hline Tertiary & 24 & 6.12 \\
\hline \multicolumn{3}{|l|}{ Religion } \\
\hline Catholic & 129 & 32.91 \\
\hline Protestant & 170 & 43.37 \\
\hline Others $^{2}$ & 93 & 23.72 \\
\hline
\end{tabular}

Mean age of respondents $=35.87 \mathrm{SD}=11.63 \mathrm{~min}$ and $\max$ age was 15 and 80 . ${ }^{1}$ Housewife, cattle keeper, and driver, ${ }^{2}$ Orthodox, "born agains", seventh day Adventists (SDA), and Muslims.

workers, all the problems mentioned by each of them were summarized and key issues were stated. For each problem, the number of times it was mentioned by the different health care workers was tallied and the total was recorded.

2.10. Ethical Considerations. Approval was got from the Uganda National Council of Science and Technology, through Makerere University, School of Public Health, and permission was sought from the district health officer. Informed consent was also sought from the respondents of $18+$ years and care takers/parents of patients 15 to 17 years of age. Assent was sought from respondents of 15 to 17 years of age.

\section{Results}

3.1. Baseline Characteristics of the Patients Attending Health Facilities in Kamuli District, March-May 2012. The study

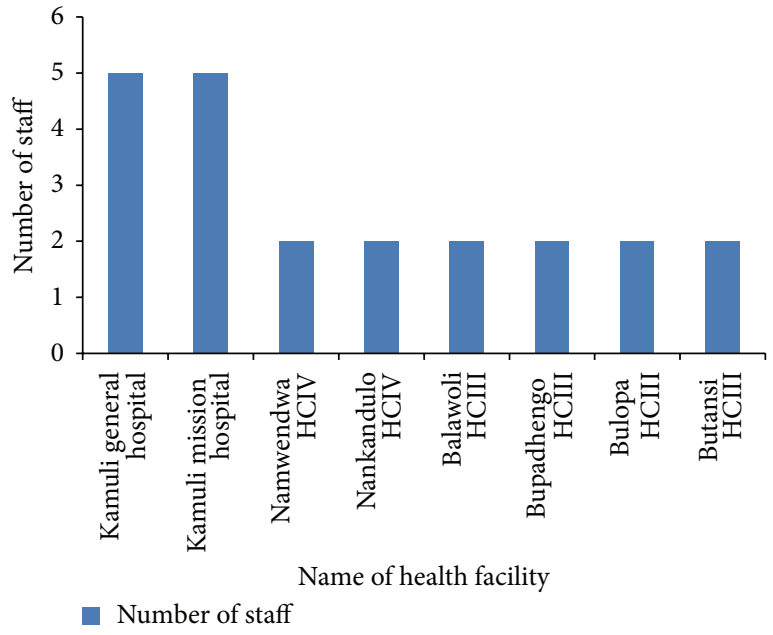

Figure 2: Laboratory staffing levels of health facilities in Kamuli district, March-May 2012.

consisted of 392 respondents of which 220 (56.12\%) were suspects, 33 (8.42\%) were newly confirmed TB cases, and $139(35.46 \%)$ were TB cases on treatment. Most of the respondents $226(58.80 \%)$ were female, belonging to age category 25-34, 123 (31.38\%), and 35-44, 128 (32.65\%), 294 (75\%) were rural residents, and $249(63.52 \%)$ were peasant farmers who had attained $191(48.72 \%)$ primary education as the highest level of education reached and the biggest numbers of the respondents were protestants (Table 2).

\subsection{Quality of the Structural Aspects of TB Service Care}

3.2.1. Structural and Overall Organizational Characteristics among the Health Facilities. All the 8 facilities had suspect, laboratory, and unit registers, intensified case finding forms, a full set of TB testing reagents, a microscope, anti-TB drugs, and a system of tracing patients who either tested positive or started on treatment and disappeared. All the 8 facilities were giving return appointments to patients who were smear negative and had been put on antibiotics at the time of study. Only $3 / 8$ facilities had the TB management desk aid, only $2 / 8$ were using and filling in the TB suspect registers consistently, and 7/8 had sputum mugs; all the 8 facilities had no evidence of their microscopes having been serviced in the last one year, $1 / 8$; that is, Nankandulo HCIV, did not have a system of identifying coughing patients from waiting areas, wards, and so on (Table 3).

3.3. Laboratory Staffing Levels of the Health Facilities. Each of the 2 HCIVs and the 4 HCIIIs had 2 laboratory personnel. Each of the hospitals only had 5 laboratory personnel (Figure 2). The most qualified laboratory staffs were technicians with a diploma in medical laboratory technology while the least qualified were microscopists who are basically on-job learners and the entomological assistants who were formerly working with the sleeping sickness control program. 
TABLE 3: Structural and overall organizational characteristics among health facilities in Kamuli district, March-May 2012.

\begin{tabular}{lcc}
\hline Characteristic & $\begin{array}{c}\text { Frequency } \\
(n=8)\end{array}$ & Percentage \\
\hline TB desk aid & $3^{* * * * *}$ & 37.5 \\
$\quad$ Available & 5 & 62.5 \\
$\quad$ Not available & & \\
TB suspect register-used consistently & $2^{* * * *}$ & 25.0 \\
$\quad$ Yes & 6 & 75.0 \\
$\quad$ No & & \\
TB request forms & 6 & 75.0 \\
$\quad$ Available & $2^{* * *}$ & 25.0 \\
$\quad$ Not available & & \\
Sputum mugs & 7 & 87.5 \\
$\quad$ Available & $1^{*}$ & 12.5 \\
$\quad$ Not available & & \\
Evidence of service of microscope & 0 & 0.0 \\
$\quad$ Available & 8 & 100 \\
$\quad$ Not available & & \\
System of identifying coughing patients & & 12.5 \\
$\quad$ No & $1^{* *}$ & 87.5 \\
Yes & 7 &
\end{tabular}

${ }^{*}$ Bupadhengo HCIII, ${ }^{* *}$ Nankandulo HCIV, ${ }^{* * *}$ Bulopa HCIII and Bupadhengo HCIII, ${ }^{* * * *}$ Bupadhengo HCIII and Butansi HCIII, ${ }^{* * * * *}$ Balawoli HCIII, Kamuli General Hospital, and Kamuli Mission Hospital.

3.4. General Perception of Interviewed Staff on Staffing Levels and Supervision among the Health Facilities. All the 20 health workers interviewed perceived the number of staff not enough to cover the TB work load and the frequency of supervision was said to be on a monthly basis by the majority of health workers $(17 / 20)$.

\subsection{Quality of the Processes of TB Service Care}

3.5.1. Patient Reported Process Characteristics of TB Care at the Health Facilities. Forty-nine (12.53\%) and 40 (10.23\%) of the patients got to know about the existence of TB services from friends/relatives/someone who had ever used the services and from the media, respectively; $52(13.27 \%)$ patients were not informed that they would transmit TB to others, 94 (23.98\%) were not informed how to stop spreading TB to others, 55 (31.98\%) patients on treatment and newly confirmed cases were not informed of the side effects of the TB drugs, 150 $(87.21 \%)$ of patients who had been started on treatment and the newly confirmed were not aware of all the signs to stop TB medication, 100 (25.51\%) received laboratory results after a period of 3-5 working days and 57 (33.14\%) patients who tested positive for TB received their drugs after a period of 2 to 5 working days (Table 4).

3.6. Number of ZN Tests Performed by Laboratories for Suspects Captured in the Year 2010. Out of the total 1453 suspects identified in the year 2010, we had 1384 (95.3\%)

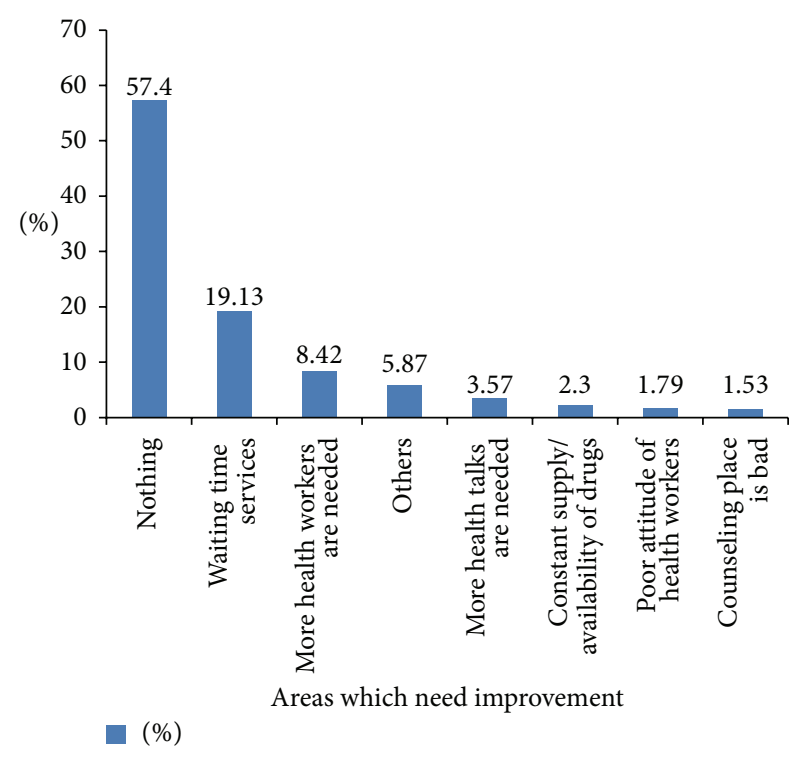

Figure 3: Challenges which need to be improved upon as reported by patients in Kamuli district health facilities, March-May 2012. Note: others included: need for food, wheel chairs, out-patient department needs improvement, improve on the infrastructure, and reduce the quantity of drugs.

microscopically being assessed by the health facilities. Out of the 1384 , we had 1115 (76.7\%) suspects having had all the two recommended smears done. However, 271 (18.5\%) suspects had only one smear done. Out of the 1453 suspects, 69 (4.7\%) who were supposed to have had microscopy done on their sputum did not have it done at all (Table 5).

3.7. Challenges Which Need to Be Improved upon as Reported by Patients and Health Care Providers. Waiting times to receive services $(19.13 \%)$, need for more workers $(8.42 \%)$, and health talks (3.57\%) are some of the key issues patients felt should be improved upon (Figure 3). The major challenges faced by the health care workers as reported by themselves were poor attitude of some health workers (mentioned 8 times), patients who default (mentioned 7 times), and fear of being infected with TB (mentioned 6 times). The other issues which providers felt should be improved upon were understaffing (mentioned 5 times), patients who do not keep appointment (mentioned 5 times), long waiting time to receive laboratory results, inadequate knowledge on $\mathrm{TB}$, limited space for TB work, and fear of the CB-DOTS program not working as expected.

Note. Frequency of challenges faced by health workers is equivalent to the number of times the challenge was mentioned by the health workers.

3.8. The Levels of TB Service Care Outcomes for New Sputum Smear Positive Cases for the Year 2010. The 8 study facilities contributed 176 new smear positive TB patients to the total number detected by the district in 2010 . Out of the 176 , $71(40.3 \%)$ patients completed their treatment, 69 (39.2\%) 
TABle 4: Patient reported process characteristics of TB care at the health facilities in Kamuli district, March-May 2012.

Process characteristic

Source of information about TB services

Referred by a health worker

Recommended by somebody who has ever used

From the media

Others

Informed that you would transmit $\mathrm{TB}$ to others

No

Yes

Informed when you stop spreading TB to others

No

Yes

Informed when next to come back for TB services

No

Yes

Informed that $\mathrm{TB}$ is cured

No

Yes

Informed about side effects of TB drugs

No

Yes

Aware of all the signs to stop TB medication

No

Yes

Informed about sputum follow-up tests at given points

No

Yes

Informed about the link between HIV and TB

No

Yes

Advised to take an HIV test

No

Yes

HW explained to you how to collect the sample

No

Yes

HWs explained things in a way you understand

No

Yes

You received all the necessary information you need to know

No

Yes

Had enough time to discuss problems with HWs

No

Yes

Opinion about attitude of staff at the health facility

Very good

Good

Fair

Poor

Frequency $(N=392)$

Percentage

294

49

40

8

52

340

94

298

64

328

24

367

55

117

150

22

12

160

24

368

25

367

63

328

16

376

54

338

110

282

127

179

84

1
75.19

12.53

10.23

13.27

86.73

23.98

76.02

16.33

83.67

6.14

93.86

31.98

68.02

87.21

12.79

6.98

93.02

6.12

93.88

6.38

93.62

16.11

83.89

4.08

95.92

13.78

86.22

28.06

71.94

32.48

45.78

21.48

0.26 
TABLE 4: Continued.

\begin{tabular}{|c|c|c|}
\hline Process characteristic & Frequency $(N=392)$ & Percentage \\
\hline \multicolumn{3}{|c|}{ Time spent to receive lab results after handing in second sample } \\
\hline $0-2$ working days & 270 & 68.88 \\
\hline 3-5 working days & 100 & 25.51 \\
\hline more than five working days & 22 & 5.61 \\
\hline \multicolumn{3}{|c|}{ Treatment observer checking on your daily intake of drugs } \\
\hline No & 23 & 16.55 \\
\hline Yes & 116 & 83.45 \\
\hline \multicolumn{3}{|c|}{ Waiting time to see the health care worker } \\
\hline Less than 1 hour & 162 & 41.33 \\
\hline 1 hour to 2 hours & 188 & 47.96 \\
\hline More than two hours & 42 & 10.71 \\
\hline \multicolumn{3}{|c|}{ Waiting time to receive anti-TB drugs } \\
\hline $0-1$ day & 115 & 66.86 \\
\hline 2 working days & 39 & 22.67 \\
\hline 3 working days & 12 & 6.98 \\
\hline 5 working days & 6 & 3.49 \\
\hline
\end{tabular}

Note: Only the newly confirmed TB cases and TB cases on treatment were asked whether they had been told about the side effects of TB drugs, and whether they knew all the signs to stop TB medication (severe skin itching, change of eye colour, impaired vision, and severe vomiting), and whether they had been informed about follow up tests of TB at different points during the course of treatment $(n=172)$.

Note: Only the TB cases on treatment were asked whether they had a treatment observer checking on their daily intake of anti-TB drugs $(n=139)$.

Note: Only the newly confirmed TB cases and TB cases on treatment were asked how long they waited to receive anti-TB drugs $(n=172)$.

Note: Waiting time to receive anti-TB drugs was considered from the time of receipt of laboratory results.

Note: Waiting time to see the health care workers was considered from the time a patient reached the facility to seeing the clinician.

TABLE 5: Number of ZN tests performed by laboratories for suspects captured in Kamuli district health facilities for the year 2010.

\begin{tabular}{|c|c|c|c|c|c|}
\hline $\begin{array}{l}\text { Name health } \\
\text { facility }\end{array}$ & Total suspects & $\begin{array}{c}\text { Total suspects } \\
\text { assessed by the } \\
\text { lab }\end{array}$ & $\begin{array}{l}\text { Total suspects } \\
\text { with } 2 \text { smears } \\
\text { done }\end{array}$ & $\begin{array}{l}\text { Total suspects } \\
\text { with } 1 \text { smear } \\
\text { done }\end{array}$ & $\begin{array}{c}\text { Suspects with } \\
\text { no smear done } \\
\text { at all }\end{array}$ \\
\hline A (HCIII) & 20 & 17 & 13 & 4 & 3 \\
\hline B (HCIII) & 44 & 44 & 43 & 1 & 0 \\
\hline C (HCIII) & 68 & 64 & 55 & 9 & 4 \\
\hline D (HCIII) & 94 & 93 & 80 & 13 & 1 \\
\hline E (HCIV) & 202 & 201 & 167 & 34 & 1 \\
\hline $\mathrm{F}(\mathrm{HCIV})$ & 48 & 42 & 38 & 4 & 6 \\
\hline G (Hosp) & 354 & 351 & 289 & 62 & 3 \\
\hline $\mathrm{H}$ (Hosp) & 623 & 572 & 430 & 142 & 51 \\
\hline Total (\%) & 1453 & $1384(95.3)$ & $1115(76.7)$ & $269(18.5)$ & $69(4.7)$ \\
\hline
\end{tabular}

were declared cured by microscopy, $1(0.6 \%)$ was a treatment failure, $22(12.5 \%)$ defaulted treatment, and $12(6.8 \%)$ died (Table 6).

\subsection{General Quality Performance of Kamuli District Health} Facilities as Judged against Guidelines/Indicators. Facility $\mathrm{H}$ (Hospital) and G (Hospital) were the worst performing facilities followed by D (HCIII). The best performance facilities were C (HCIII) and B (HCIII) (Table 7).

Though we did not have data on patients' views for $\mathrm{F}$ (HCIV) to allow scoring of certain quality parameters like waiting time to receive laboratory results, anti-TB drugs, patients awareness on adverse effects of drugs, and advise to test for HIV, we still went ahead to score the rest of the parameters whose data was available and finally concluded on the facilities performance. For the few parameters assessed, the score was already high, so we concluded that the facility's quality of TB service care was poor.

The worst performance indicators were making sure that every suspect is tested for TB with at least one ZN test. That was followed by ensuring two ZN tests for every suspect and the number cured whose percentage was very low (Table 7).

Note. For more information on how the scores were generated and what they meant, check Table 1: performance indicators/guidelines against which quality for Kamuli district health facilities was judged. 
TABLE 6: Outcomes of TB service care for new sputum smear positive cases in Kamuli district health facilities for the year 2010.

\begin{tabular}{|c|c|c|c|c|c|c|c|c|c|}
\hline $\begin{array}{l}\text { Name of } \\
\text { health } \\
\text { facility }\end{array}$ & $\begin{array}{c}\text { Number of } \\
\text { smear } \\
\text { positives } \\
\text { identified in } \\
2010 \\
\end{array}$ & $\begin{array}{l}\text { Number } \\
\text { started on } \\
\text { treatment }\end{array}$ & $\begin{array}{c}\text { Number on } \\
\text { F-DOTS }\end{array}$ & $\begin{array}{c}\text { Number } \\
\text { on } \\
\text { CB-DOTS }\end{array}$ & $\begin{array}{l}\text { Completed } \\
\text { treatment }\end{array}$ & Cured & $\begin{array}{l}\text { Treatment } \\
\text { failures }\end{array}$ & $\begin{array}{l}\text { Defaulted } \\
\text { treatment }\end{array}$ & Died \\
\hline A (HCIII) & 01 & 01 & - & - & 01 & - & - & - & - \\
\hline B (HCIII) & 03 & 03 & - & 03 & 01 & 02 & - & - & - \\
\hline C (HCIII) & 08 & 08 & - & 08 & 03 & 04 & - & 01 & - \\
\hline D (HCIII) & 13 & 13 & - & 11 & 09 & 03 & - & - & 01 \\
\hline E (HCIV) & 18 & 18 & 01 & 17 & 08 & 06 & - & 02 & 02 \\
\hline $\mathrm{F}(\mathrm{HCIV})$ & 02 & 02 & - & 01 & 02 & - & - & - & - \\
\hline G (Hospital) & 72 & 72 & 19 & 21 & 28 & 030 & - & 11 & 03 \\
\hline H (Hospital) & 59 & 59 & - & 09 & 19 & 024 & 01 & 08 & 06 \\
\hline Total (\%) & 176 & 176 & $20(11.4)$ & $70(40)$ & $71(40.3)$ & $69(39.2)$ & $1(0.6)$ & $22(12.5)$ & $12(6.8)$ \\
\hline
\end{tabular}

F-DOTS-facility-directly observed therapy-short course, CB-DOTS-community based-directly observed therapy-short course.

TABLE 7: General quality performance of Kamuli district health facilities as judged against guidelines/indicators.

\begin{tabular}{lcccccccccccccccc}
\hline Health facility & A & B & C & D & E & F & G & H & I & J & K & L & M & N & O & Total score \\
\hline H (Hospital) & 2 & 1 & 2 & 2 & 1 & 1 & 3 & 2 & 2 & 2 & 1 & 2 & 2 & 3 & 3 & $\mathbf{2 8}$ \\
G (Hospital) & 2 & 1 & 2 & 2 & 1 & 1 & 2 & 2 & 3 & 1 & 1 & 2 & 1 & 3 & 2 & $\mathbf{2 6}$ \\
F (HCIV) & 2 & - & - & 2 & 2 & 2 & 3 & 2 & - & - & 2 & 2 & 1 & 1 & 1 & $\mathbf{2 0}$ \\
E (HCIV) & 1 & 1 & 1 & 2 & 1 & 1 & 2 & 1 & 3 & 1 & 1 & 2 & 1 & 2 & 2 & $\mathbf{2 2}$ \\
D (HCIII) & 2 & 2 & 1 & 2 & 1 & 1 & 3 & 2 & 1 & 1 & 1 & 2 & 1 & 2 & 2 & $\mathbf{2 4}$ \\
C (HCIII) & 2 & 1 & 1 & 2 & 1 & 1 & 2 & 1 & 2 & 1 & 1 & 2 & 1 & 1 & 1 & $\mathbf{2 0}$ \\
B (HCIII) & 2 & 1 & 1 & 2 & 1 & 1 & 1 & 1 & 3 & 1 & 1 & 2 & 1 & 1 & 1 \\
A (HCIII) & 2 & 1 & 1 & 2 & 1 & 1 & 2 & 2 & 2 & 1 & 1 & 2 & 1 & 1 & 1 & $\mathbf{2 0}$ \\
\hline Total score & $\mathbf{1 5}$ & $\mathbf{8}$ & $\mathbf{9}$ & $\mathbf{1 6}$ & $\mathbf{9}$ & $\mathbf{9}$ & $\mathbf{1 8}$ & $\mathbf{1 3}$ & $\mathbf{1 6}$ & $\mathbf{8}$ & $\mathbf{9}$ & $\mathbf{1 6}$ & $\mathbf{9}$ & $\mathbf{1 4}$ & $\mathbf{1 3}$ \\
\hline
\end{tabular}

Note: for both the health facilities and guidelines, least performance score implied good quality services while the greatest score implied poor quality services offered by a facility.

Key:

a-every staff participates on identification of tuberculosis cases, B-waiting to receive laboratory result, C-waiting time to receive treatment, D-two ZN tests done for every suspect, E-system of identifying coughing clients from waiting, areas, and other places, F-system of tracing lost clients, G-assessing every patient with at least one test, H-is every TB client on DOTs? I-TB patients aware of adverse effects/side effects of TB drugs, J-every suspect advised to test, for HIV, K-cases notified, L-number of patients cured, $\mathrm{M}$-number of treatment failures, $\mathrm{N}$-number of patients who defaulted, O-number of patients who died.

The patients overall perception of the services together with the WHO, NTLP, International standards for tuberculosis care (ISTC), and the researchers' own set perception about the quality of services offered were used to judge the overall quality of services offered at the facilities/level of execution of the different indicators.

\section{Discussion}

In this cross-sectional study conducted to assess quality of TB health services in a rural district, we found that the patients were not receiving adequate information about TB from the health providers. This is evidenced by a number of patients who were not informed about the possibility of transmitting $\mathrm{TB}$ to others, how to stop transmitting $\mathrm{TB}$, the side effects of TB drugs, and signs of side effects in order to stop TB medications. Limited information among the patients could explain the poor outcomes such as the high default and deaths rates reported in the study. Health care providers often try to supply information to patients and to motivate them and recognize the importance of behavioral skills in improving health. However, there is evidence that, in practice, health providers are constrained by time and hence they give limited information [6]. In order to solve the problem of inadequate information, the TB program could develop a standardized approach to patient health education where the health providers use structured guidelines and also provide user-friendly translated information, education, and communication (IEC) materials including visual aids to all TB patients. A systematic review showed that overall education or counseling interventions may increase successful treatment completion although the magnitude of benefit is likely to vary depending on the nature of the intervention and the setting [7].

Patient waiting time to receive services is one of the major challenges hindering TB control as evidenced by a 
number of patients who received laboratory results after a period ranging from three to more than five working days. The National TB Reference Laboratory standard operating Procedure states that microscopy results should be reported within 24 hours; anything beyond 24 hours is considered unacceptable.

According to the ministry of health guidelines, a patient is supposed to be started on treatment as soon as they are identified to have TB; yet the findings reveal a number of patients taking days without treatment initiation. Gulu Regional Referral Hospital project also revealed that over 50\% of patients at Gulu TB Ward clinic in 2008 had a waiting time of 7 days from TB suspicion to treatment initiation [8]. Another study carried out in Botswana, on completeness and timeliness of treatment initiation after laboratory diagnosis of tuberculosis, showed that $11.8 \%$ patients had a delay in treatment initiation [9]. In Uganda and much of Africa, the TB patient load is increasing due to the underlying HIV epidemic that partly fuels the TB epidemic. Moreover, there is a shortage of skilled healthcare personnel to deliver health services leading to an overstretched health system $[10,11]$. In order to improve the workflow and mitigate the waiting problems innovative ways of delivery of care such as task-shifting through training a cadre of lay health workers to perform simple tasks like collecting sputum and initial patient registration could be explored. Service care programs for HIV/AIDS have employed similar low-cost models of task-shifting and they have helped to relieve the strain on the health system $[12,13]$.

Adherence to recommended guidelines for smear microscopy was very low indicating poor performance almost across all health facilities. The study reveals $18.5 \%$, and $4.7 \%$ of the TB suspects not having had a second smear and not having microscopy done at all instead of the two tests recommended by the guidelines. This highlights an important gap in the processes that could be leading to poor quality of service due to improper management of the patients and may perhaps imply lack of competence on the providers' side. Similarly, a study carried out in Botswana, on completeness and timeliness of treatment initiation after laboratory diagnosis of tuberculosis, also revealed that $10.2 \%$ patients had only one smear done [9].

Most of the TB patients on treatment had a treatment observer checking and ensuring on their daily intake of drugs, the NTLP recommends every patient to have a treatment observer. According to one of the health care workers in facility E (HCIV), the health care workers are not sure whether what they refer to as DOTS is actually working and they are not sure of whether the treatment observers are carrying out their role. Verification of such a finding using a community based study is needed for the government to be sure whether the DOTS programme is working as expected and yielding positive results. This doubt was also cited by a paper from Ethiopia, which recommended for an expanded community-based study to better guide quality DOTS programs. The number of patients declared cured is still wanting as seen from the outcome table; this actually means that patients just take medication but the issue of follow ups recommended at 2, 5, and 8 months of treatment is not being ensured by health facilities. The flow of patients and the location of the unit TB register need to be checked and organized so that no patient gets treatment without a followup test.

The study revealed a high proportion of treatment defaulters. This could be explained by the patients not getting adequate information about TB and a failing DOTS program. According to a qualitative study carried out in Norway on barriers and facilitators of adherence to TB treatment in patients on concomitant TB and HIV treatment, some of the barriers to treatment adherence were experiencing side effects, economic constraints, lack of food, and lack of adequate communication with health professionals among other factors [14].

All the health facilities had the basic essential requirements for TB diagnosis and treatment. This could be attributed to consistent monthly and quarterly supervisions by the district team, and a few times by the ministry of health officials as recommended by the ministry of health and presence of development and implementing partners like global fund, STAR-EC, and Plan-Uganda could also be reason for the essential requirements being in place. A study carried out on quality of tuberculosis care in Ethiopia also revealed delivery of materials, drugs, and supplies for tuberculosis control activities being fairly good [15].

All the facilities had the TB suspect register but only two were consistently using and filling the register. According to the guidelines, all the TB suspects identified in the health facility are supposed to be registered in the TB suspect register and are then sent to the laboratory for diagnosis. This could be attributed to health workers not knowing the purpose of such a register. According to the NTLPUganda, the suspect register provides a means of recording all symptomatic patients classified as suspects; it includes all patients who have a cough for two weeks or more. The suspect register is a useful tool to monitor sputa sent to the laboratory, for evaluating case detection and determining the prevalence of TB suspects at facility level. It is also useful for estimating supply levels needed for bacteriologic exams. The National TB and Leprosy Programme should think of evaluating the usage and filling of the suspect register in other districts before coming up with a conclusive way forward.

Our study points out to poor attitude of fellow health workers and fear of being infected with $\mathrm{TB}$ as some of the key challenges faced by health workers. Similarly, a cross-sectional study carried out in Ghana also pointed out that attitudes of healthcare workers towards TB patients and health staff's own fear of TB were some of the major problems hindering TB work and accelerating stigma [16]. The programme should place emphasis on participatory inservice training for health workers focusing on patientcentered continuous quality improvement, teamwork and, implementation of infection control practices. A similar approach to quality improvement was used in a TB program in Cape Town, South Africa [17].

The main limitations in our study were patients' exit interviews using a cross-sectional design; this has inherent 
potential for several biases such as social desirability bias and selection bias. The selection bias was minimized by sampling from different levels of health facilities and the social desirability bias was reduced by using volunteers who are not health workers to conduct the patient interviews. Since some of the results are from secondary TB program data that was not collected primarily for research, the potential for some inaccuracy cannot be ruled out. However, that was minimized by verification of data using the district TB registers, consulting the district TB leprosy focal person, and the various health facility staff met. Gaps in data due to missing information on some indicators and difficulty in accessing some registers could have compromised the quality of the record review results. Recalling accurately the presence of some items like reagents and drugs in the last one year may have compromised the study findings, but that was minimized by use of stock cards to verify the responses given. Despite the highlighted limitations, the study's greatest strength was the use of mixed methods, quantitative and qualitative methods of data collection to examine the complex aspects of quality of TB services and to provide some initial evidence that can be used to improve patient care.

\section{Conclusion}

The basic structural essential requirements for TB diagnosis and treatment were in place. However, the patients were not receiving adequate information about $\mathrm{TB}$ from the health workers, long patient waiting time to receive services at each of the levels of care, poor attitude of fellow health workers and health workers' own fear of being infected with $\mathrm{TB}$, and the low percentage of cure are some of the key process issues hindering TB control in the rural setting. Comprehensive strengthening of the health system focusing on quality of support supervisions, patient follow up and promoting infection control measures and increasing health staffing levels at the health facilities are crucial.

\section{Disclaimer}

The findings and conclusions in this report are those of the authors and do not necessarily represent the official position of the Uganda National Tuberculosis and Leprosy Programme (NTLP)/National Tuberculosis Reference Laboratory (NTRL).

\section{Conflict of Interests}

None of the authors have commercial or other financial interests associated with the information presented in this paper.

\section{Acknowledgments}

The authors acknowledge the National Tuberculosis and Leprosy Programme and National Tuberculosis and Reference Laboratory where they got a lot of information concerning the subject of study. They also appreciate the assistance provided by the district tuberculosis and leprosy supervisor Mr. Mpata Samuel and the research assistants.

\section{References}

[1] WHO, Global Tuberculosis Control Report, 2013.

[2] A. Donabedian, "Evaluating the quality of medical care," Milbank Quarterly, vol. 83, no. 4, pp. 691-729, 2005.

[3] S. M. Campbell, M. O. Roland, and S. A. Buetow, "Defining quality of care," Social Science and Medicine, vol. 51, no. 11, pp. 1611-1625, 2000.

[4] MoH-Uganda, The Uganda TB Communication Strategy, National Tuberculosis \& Leprosy control Programme \& Health Promotion and Education Division, Kampala, Uganda, 2008.

[5] N. T. L. P. MoH-Uganda, Tuberculosis Case Management Desk Aide, 2007.

[6] H. Waitzkin and J. D. Stoeckle, "Information control and the micropolitics of health care: summary of an ongoing research project," Social Science and Medicine, vol. 10, no. 6, pp. 263-276, 1976.

[7] J. M. M’imunya, T. Kredo, and J. Volmink, "Patient education and counselling for promoting adherence to treatment for tuberculosis," Cochrane Database of Systematic Reviews, vol. 5, p. CD006591, 2012.

[8] S. R. L. Angee, B. Sheila, A. Yovention, and N. Christine, Reducing Patient Waiting Time to Initiate Tuberculosis Treatment at Gulu, Gulu Regional Referral Hospital, Makerere University College of Health Sciences, Institute of Public Health, Kampala, Uganda, 2010.

[9] T. L. Creek, S. Lockman, T. A. Kenyon et al., "Completeness and timeliness of treatment initiation after laboratory diagnosis of tuberculosis in Gaborone, Botswana," International Journal of Tuberculosis and Lung Disease, vol. 4, no. 10, pp. 956-961, 2000.

[10] S. Naicker, J. Plange-Rhule, R. C. Tutt, and J. B. Eastwood, "Shortage of healthcare workers in developing countriesAfrica," Ethnicity \& Disease, vol. 19, no. 1, supplement 1, pp. S1S61, 2009.

[11] A. Chikanda, "Skilled health professionals' migration and its impact on health delivery in Zimbabwe," 2004.

[12] R. Zachariah, N. Ford, M. Philips, M. Massaquoi, V. Janssens, and A. D. Harries, "Task shifting in HIV/AIDS: opportunities, challenges and proposed actions for sub-Saharan Africa," Transactions of the Royal Society of Tropical Medicine and Hygiene, vol. 103, no. 6, pp. 549-558, 2009.

[13] M. Callaghan, N. Ford, and H. Schneider, "A systematic review of task- shifting for HIV treatment and care in Africa," Human Resources for Health, vol. 8, no. 1, article 8, 2010.

[14] M. K. Gebremariam, G. A. Bjune, and J. C. Frich, "Barriers and facilitators of adherence to $\mathrm{TB}$ treatment in patients on concomitant TB and HIV treatment: a qualitative study," BMC Public Health, vol. 10, article 651, 2010.

[15] A. Girma, D. Mariam, and K. Deribe, "Quality of tuberculosis care in six health facilities of Afar Region, Ethiopia," Ethiopian Medical Journal, vol. 48, no. 3, pp. 195-202, 2010.

[16] Dodor and E. Atsu, An exploration of the causes, manifestations and consequences of tuberculosis stigma in an urban district in Ghana [Ph.D. thesis], 2009.

[17] S. Lewin, J. Dick, M. Zwarenstein, and C. J. Lombard, "Staff training and ambulatory tuberculosis treatment outcomes: a cluster randomized controlled trial in South Africa," Bulletin of the World Health Organization, vol. 83, no. 4, pp. 250-259, 2005. 


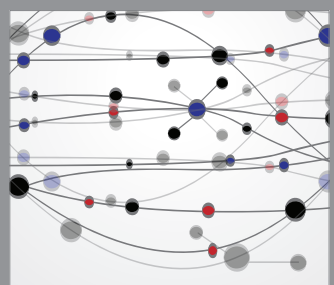

The Scientific World Journal
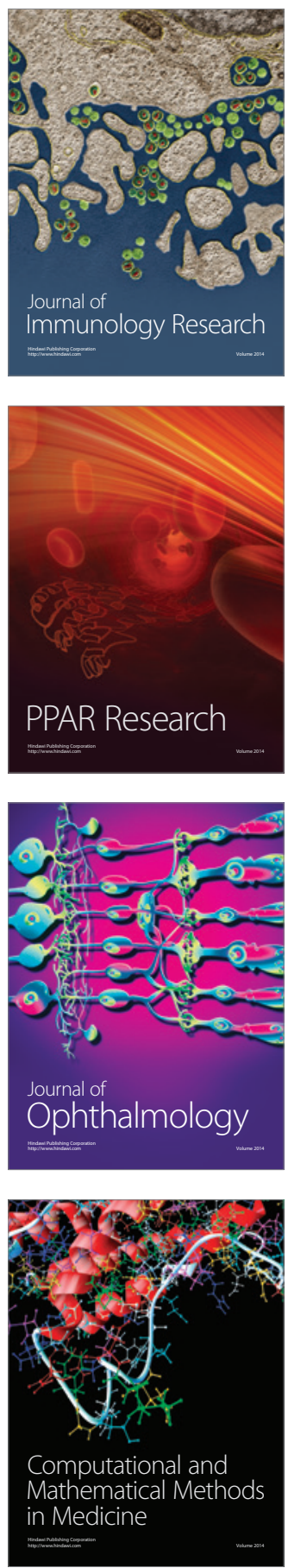

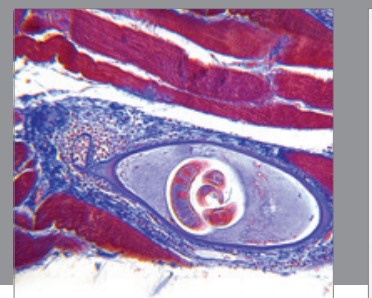

Gastroenterology

Research and Practice
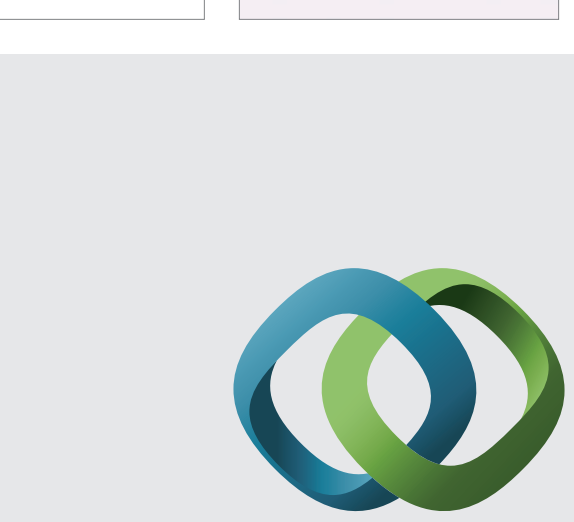

\section{Hindawi}

Submit your manuscripts at

http://www.hindawi.com
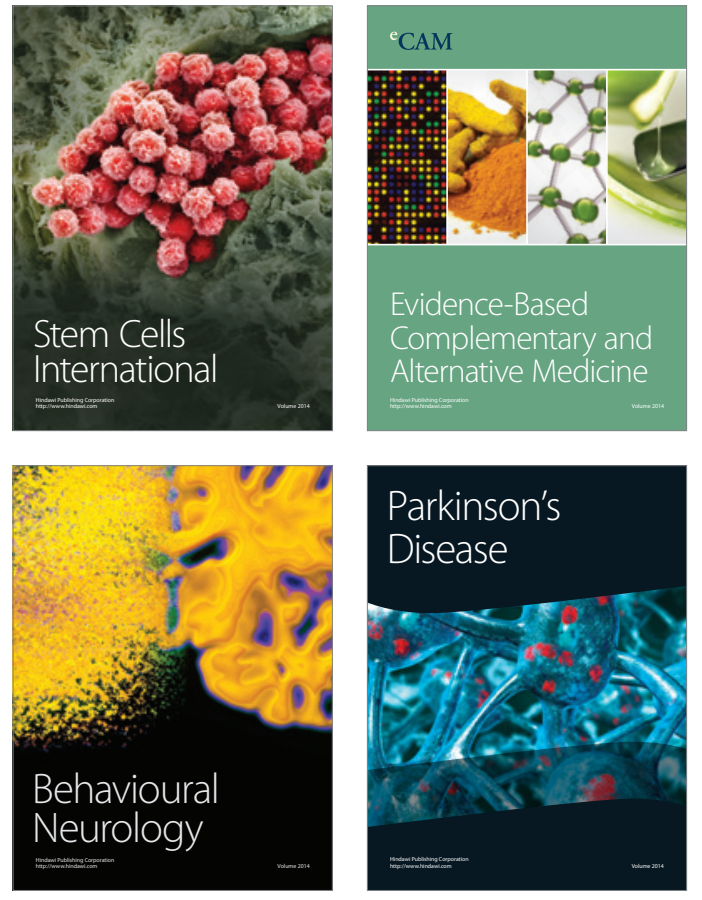
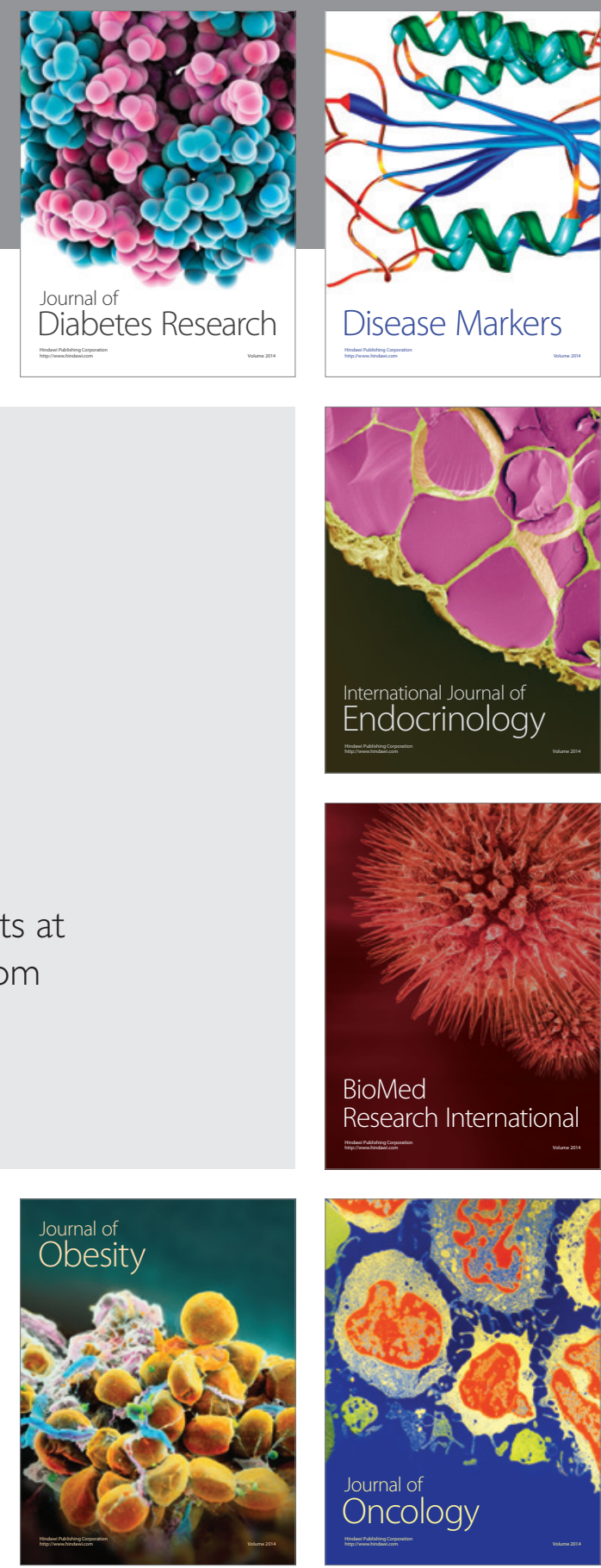

Disease Markers
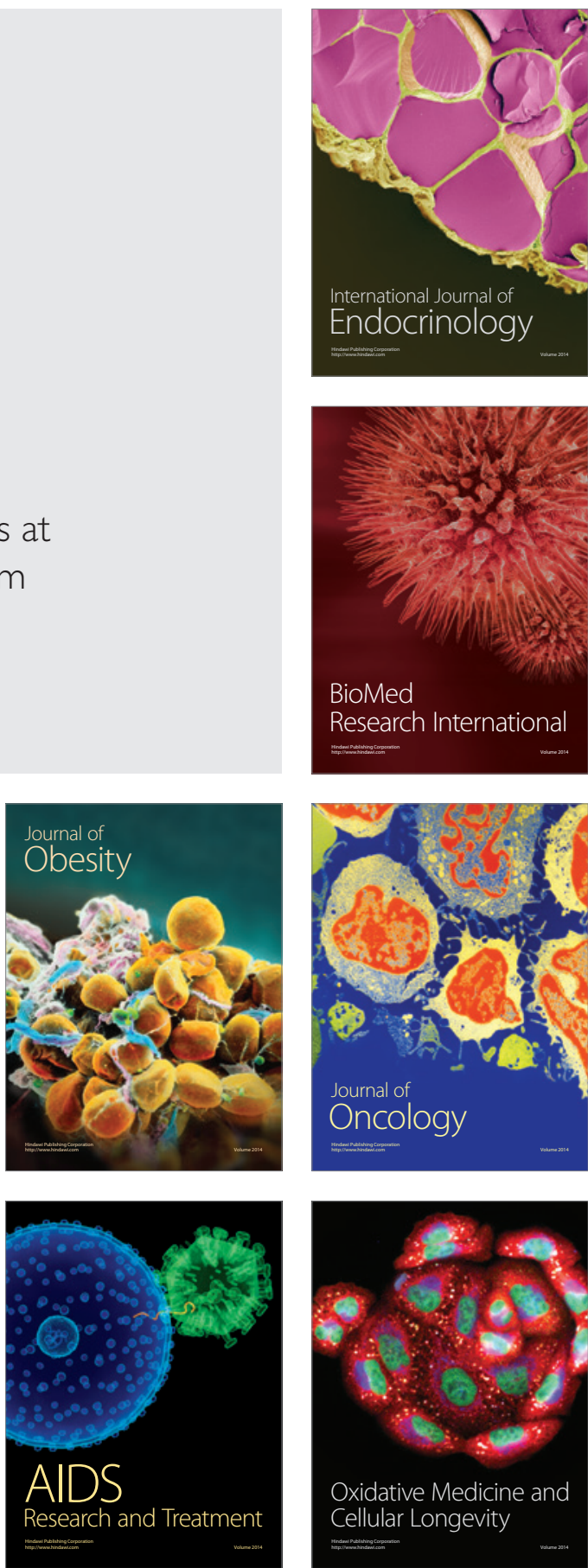